\title{
Ocean bottom geoacoustic characterization using surface ship noise of opportunity
}

\author{
Qun-yan Ren*† and Jean-Pierre Hermand* \\ *Environmental Hydroacoustics lab, Université libre de Bruxelles (U.L.B.) \\ av. F. D. Roosevelt 50, CP 194/05, B-1050 Brussels, Belgium \\ ${ }^{\dagger}$ National Laboratory of Underwater Acoustic Technology, Harbin Engineering University \\ Harbin, Heilongjiang, 150001, China
}

\begin{abstract}
The broadband noise field of a ship of opportunity often exhibits environment dependent striation structure in the frequency-range plane. For the soft-layered sediment environment studied in this paper, the striation structure is critically determined by sub-bottom sound speed $\left(C_{b o t}\right)$, sediment thickness $(H)$ and sediment sound speed $\left(C_{s e d}\right)$. Numerical simulations demonstrate that striations in different frequency bands have different sensitivities to the three critical parameters. The sensitivity differences are used here to progressively estimate the $C_{b o t}$, $H$ and $C_{\text {sed }}$. We first use low-frequency striation structure to estimate the $C_{b o t}$, then obtain a preliminary estimation of the $H$ and $C_{s e d}$ with a set of low-frequency striations, and finally find the best-fit solutions from previous estimates using high frequency striation structure. We processed passive ship run data collected in Mediterranean Sea in 2007. The good agreement between our results with active inversion methods demonstrates the accuracy of the method for ocean bottom geoacoustic characterization.
\end{abstract}

\section{INTRODUCTION}

The ocean bottom geoacoustic properties and their spatial distributions are greatly required for shallow-water sound propagation prediction and relative sonar applications. A controlled source is usually used in, e.g., direct measurements [1], linear and nonlinear inversion methods [2]-[4], analytical or numerical adjoint models [5] to measure the geoacoustic properties. The high maintenance cost makes high power source limited for some situations. In the view of environmental protection, it is better to benefit from sources already present in the ocean [6]-[9].

In coastal regions, the noise field between tens and hundreds $\mathrm{Hz}$ is mainly due to shipping activities, which is a good resource for underwater acoustic applications in these areas. The researches on geoacoustic inversion using ship noise have already been conducted both in frequency domain [10]-[14] and time domain [15], [16]. Morley et al [17] gave some notes on geoacoustic inversion using ship noise with a towed array.

The ship noise spectra and level is greatly dependent on its navigation speed, sea state, course, SSP in water column, but its frequency-range distribution usually exhibits stable striations, which is favourable for passive acoustic applications.

Work supported by Belgian National Fund for Scientific Research (F.N.R.S.) with the 'Aspirant' grant, Office of Naval Research grant N0001407-1-1069, National Nature Science Foundation of China grant 50979019, European Seas Observatory Network (ESONET) Network of Excellence and Ocean Acoustic Exploration IRSES (OAEx).
The waveguide invariant theory [18] provides an interpretation of the striation slope, based on which many applications are developed, especially for inverse problems [12], [20]-[24]. In this paper, the use of local striation features for ocean bottom geoacoustic characterization is emphasised. A multi-scale line filter [25] is briefly introduced to process the interference structure for striation extraction.

For the particular soft layered sediment environment, the sub-bottom sound speed $\left(C_{b o t}\right)$, sediment thickness $(H)$ and sediment sound speed $\left(C_{s e d}\right)$ have determined effects on the striation structure [30]. Furthermore, we find the $C_{b o t}, H$ and $C_{\text {sed }}$ have different degree effects on different frequency-band striations by numerical simulations. The different sensitivities are used here to progressively invert for these three parameters.

Subsequent sections of the paper are organized as follows: The image processing method used in this paper is presented in Sec. II. Section III introduces some theoretical basis of our method. The passive ship acoustic run experiment is described in Sec. IV. Sec. V is data processing for ocean bottom geoacoustic characterization. Conclusions are given in Sec. VI.

\section{INTERFERENCE STRUCTURE PROCESSING FOR STRIATION EXTRACTION}

We applied a 2D multi-scale line filter [25] to extract the striations from the broadband ship noise. This filter analyzes the eigenvlaues of an image Hessian matrix calculated at different scales $\sigma$ [26], and outputs maximum response at the scale that best match the linear structure width in the image:

$$
M=\max _{\sigma_{\min } \leq \sigma \leq \sigma_{\max }} M(\sigma)
$$

and

$$
M(\sigma)=\left\{\begin{array}{lr}
0 & \text { if } \lambda_{2}<0 \\
\exp \left(-\frac{R_{\sigma}^{2}}{2 \beta^{2}}\right)\left(1-\exp \left(-\frac{S_{\sigma}^{2}}{2 \gamma^{2}}\right)\right)
\end{array}\right.
$$

where

$\sigma_{\min }$ and $\sigma_{\max }$ are the minimum and maximum of the scales between which relevant linear structures are expected to be found;

$R$ is the geometric ratio that ensures only the geometric information of the image is used; 


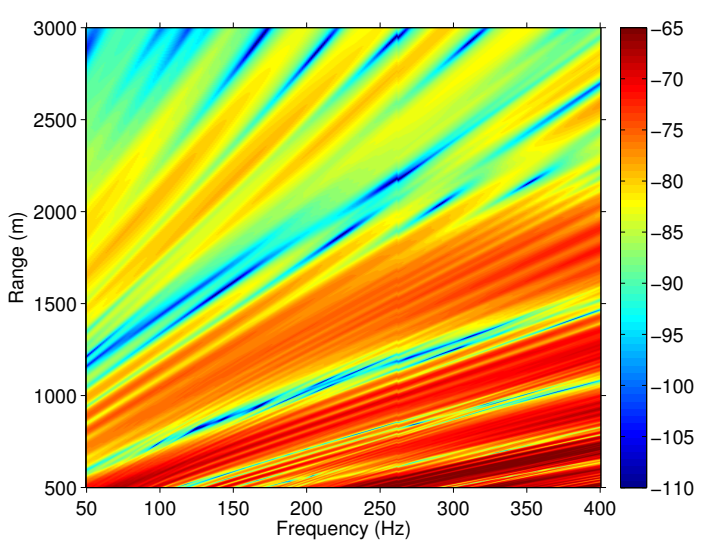

(a)

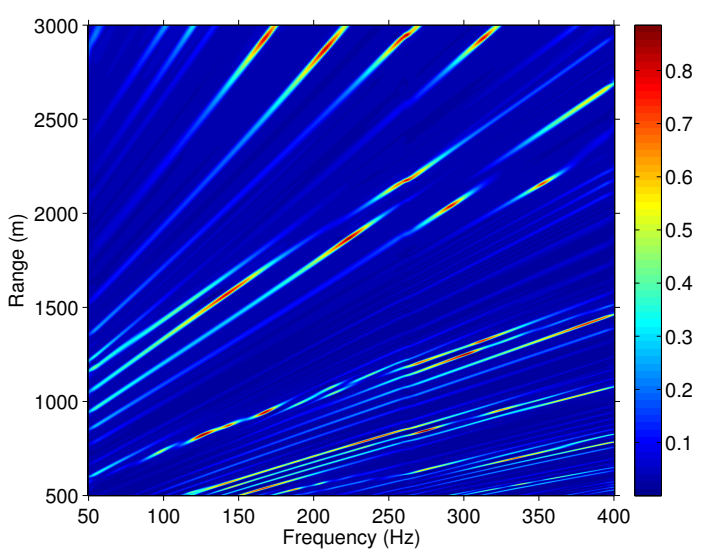

(b)

Figure 1: (a) Predicted sound interference structure for the Yellow Shark environment [4] (in $\mathrm{dB}$ ) and (b) its filtered version by the multi-line filter for scale intervals $[1,3]$ with an increment of 1 .

$S$ is the SNR, measuring the contrast of the analyzed regions with that of background;

$\beta$ and $\gamma$ are the thresholds that controlling the sensitivities of $R_{\sigma}$ and $S_{\sigma}$, and they are respective set to 1.0 and 15 in this paper.

Figure 1(a) is the simulated sound interference structure based on the Yellow Shark environment (environmental parameters are given in Tab. I) calculated by Kraken-C [27]. The source and receiver depths are set to $3.5 \mathrm{~m}$ and $20 \mathrm{~m}$, respectively. Striations with different widths are visible in this figure. Figure 1(b) is filtered result of Fig. 1(a) by setting $\sigma_{\min }$ and $\sigma_{\max }$ respectively as 1 and 3, with an increment of 1. Most striations are detected and isolated, especially for low frequency.

\section{THEORETICAL BASIS}

For an hypothetical environment $E_{0}$, the scalar sound intensity $I$ generated by an omni-directional point source of circular frequency $\omega$ at depth $z_{0}$, received at range $r_{0}$ and depth $z$, can be expressed as a summation of finite number of normal
Table I: Yellow Shark environmental parameters [4].

\begin{tabular}{l|l|c}
\hline Water column & depth & $113.1 \mathrm{~m}$ \\
\hline \multirow{4}{*}{ Sediment } & thickness & $7.5 \mathrm{~m}$ \\
\cline { 2 - 3 } & density & $1.5 \mathrm{~kg} / \mathrm{m}^{3}$ \\
\cline { 2 - 3 } & compression speed (top layer) & $1470 \mathrm{~m} / \mathrm{s}$ \\
\cline { 2 - 3 } & compression speed (bottom layer) & $1485 \mathrm{~m} / \mathrm{s}$ \\
\cline { 2 - 3 } & attenuation & $0.06 \mathrm{~dB} / \lambda$ \\
\hline \multirow{3}{*}{ Halfspace } & density & $1.8 \mathrm{~kg} / \mathrm{m}^{3}$ \\
\cline { 2 - 3 } & compression speed & $1530 \mathrm{~m} / \mathrm{s}$ \\
\cline { 2 - 3 } & attenuation & $0.15 \mathrm{~dB} / \lambda$ \\
\hline
\end{tabular}

modes [28]:

$$
\begin{aligned}
I\left(\omega, r_{0}, z, E_{0}\right) & \propto \\
\frac{1}{r_{0}} \sum_{l}^{N} B_{l}^{2} & +\frac{1}{r_{0}} \sum_{l, m(l \neq m)}^{N} B_{l} B_{m}^{*} \cos \left(\Delta \xi_{l, m} r_{0}\right),
\end{aligned}
$$

where $N$ is the number of received propagation modes, $B_{l}=$ $\sqrt{2 \pi / k_{l}} \phi_{l}\left(z_{0}\right) \phi_{l}(z), \phi_{l}$ and $\xi_{l}$ are respectively the modal function and eigenvalue for mode $l, \Delta \xi_{l, m}=\xi_{l}-\xi_{m}$, and $*$ denotes complex conjugate.

The scalar intensity consisting of two terms: incoherent term and coherent term. The striation structure is give risen by the coherent term, which is a result of mode interference. As in Eq. 3, the mode interference is characterized by the number of propagation modes, the receiver and source depths, the modal magnitude and the phase difference. Among these factors, the number of propagation mode $N$ is a critical parameter, which determines the number of mode interferences (striation number). Other factors mainly have effects on striation intervals and magnitudes.

According to the effective depth theory [29], $N$ is determined by the ratio of the sound speeds of water column and ocean bottom. In this environmental model, the $C_{\text {sed }}$ is lower than that of the water column, the acoustic signal will penetrate through the sediment layer and be reflected by the sub-bottom for long range propagation. In this particular environment, the $C_{b o t}$ virtually determines $N$ and and hence the striation structure, especially the striation number.

Figure 2 gives some numerical results for the broadband sound distributions based on the soft layered environmental model with different $C_{b o t}, H$ and $C_{\text {sed }}$ that given in Tab. II. The other parameters are the same as Tab. I. The $C_{b o t}$ has a critical effect on the striation numbers for both high and lowfrequency striations. While for a fixed $C_{b o t}$, the low-frequency striation locations change slowly with $H$ and $C_{\text {sed }}$ [30]. The high-frequency (say above $300 \mathrm{~Hz}$ ) striation structure is sensitive to all these three parameters.

\section{EXPERIMENT DESCRIPTION}

The ship noise data processed here were collected on the 23th of April, 2007. Many other measurements have been integrated to efficiently and globally characterize the underwater environment [31]-[33] of this area. The R/V Leonardo was used as source of opportunity in the passive acoustic run that 


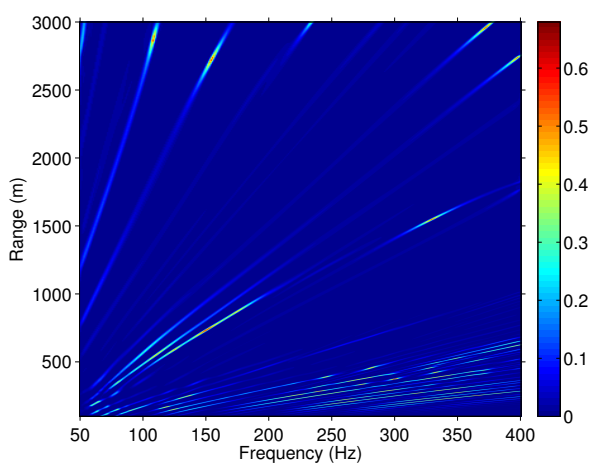

(a)

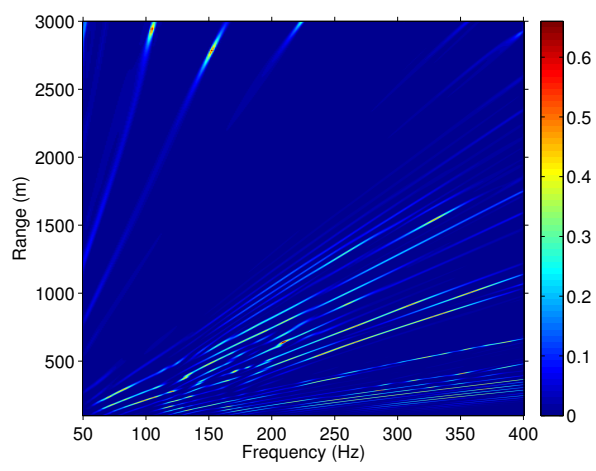

(d)

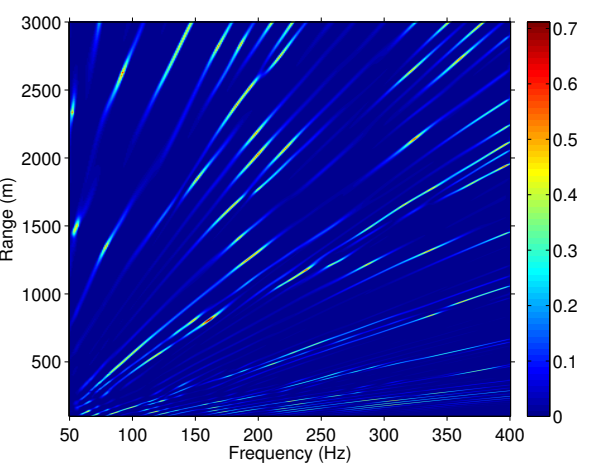

(b)

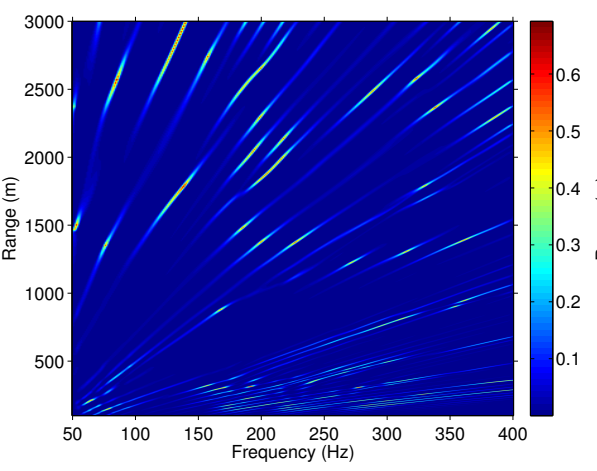

(e)

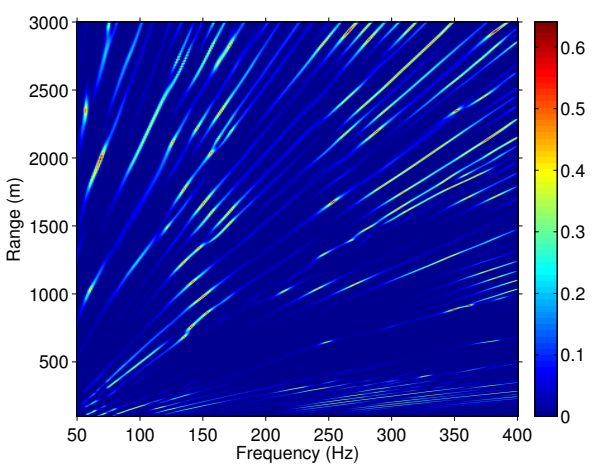

(c)

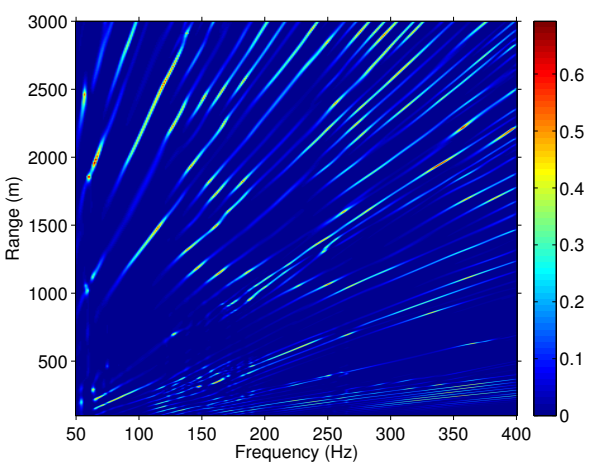

(f)

Figure 2: The predicted frequency-range sound distributions (after multi-scale line filtered) for the seabed with different $C_{b o t}$, $H$ and $C_{\text {sed }}$ : (a) $C_{b o t}=1530 \mathrm{~m} / \mathrm{s}, H=2.5 \mathrm{~m}$ and $C_{\text {sed }}=1500 \mathrm{~m} / \mathrm{s}$, (b) $C_{b o t}=1590 \mathrm{~m} / \mathrm{s}, H=0.5 \mathrm{~m}$ and $C_{\text {sed }}=1460$ $\mathrm{m} / \mathrm{s}$, (c) $C_{b o t}=1650 \mathrm{~m} / \mathrm{s}, H=2.5 \mathrm{~m}$ and $C_{s e d}=1500 \mathrm{~m} / \mathrm{s}$, (d) $C_{b o t}=1530 \mathrm{~m} / \mathrm{s}, H=5.5 \mathrm{~m}$ and $C_{\text {sed }}=1490 \mathrm{~m} / \mathrm{s}$, (e) $C_{\text {bot }}=1590 \mathrm{~m} / \mathrm{s}, H=5.5 \mathrm{~m}$ and $C_{\text {sed }}=1490 \mathrm{~m} / \mathrm{s}$ and (f) $C_{b o t}=1650 \mathrm{~m} / \mathrm{s}, H=5.5 \mathrm{~m}$ and $C_{\text {sed }}=1460 \mathrm{~m} / \mathrm{s}$.

Table II: The selected $C_{b o t}, h$ and $C_{\text {sed }}$ for the predicted interference structures in Fig. 2.

\begin{tabular}{l|l|l|l}
\hline \hline & $C_{\text {bot }}(\mathrm{m} / \mathrm{s})$ & $H(\mathrm{~m})$ & $C_{\text {sed }}(\mathrm{m} / \mathrm{s})$ \\
\hline Fig. 2(a) & 1530 & 2.5 & 1500 \\
\hline Fig. 2(b) & 1590 & 0.5 & 1460 \\
\hline Fig. 2(c) & 1650 & 2.5 & 1500 \\
\hline Fig. 2(d) & 1530 & 5.5 & 1490 \\
\hline Fig. 2(e) & 1590 & 5.5 & 1490 \\
\hline Fig. 2(f) & 1650 & 5.5 & 1460 \\
\hline \hline
\end{tabular}

denoted as L\#2. The geometry of run $\mathrm{L \# 2}$ is indicated by red line in Fig. 3. Two compact vertical ocean-acoustic arrays (OAA) were deployed from a rubber boat (RHIB). The shallow and deep arrays were configured with respectively four and five 5-m-spaced hydrophones with maximum depths of 35 $\mathrm{m}$ and $105 \mathrm{~m}$, respectively. The drift course of the OAA is marked as red dotted line.

An active measurement was also performed in this area. The sound source was deployed from R/V Leonardo (red cross) and recorded by the OAA deployed from RHIB, drifted slowly towards (yellow dished line) R/V Leonardo. The active data was processed by a sequential Bayesian filtering technique [32] and the results serve as reference to validate our results.

In this paper, the sensitivity differences are used to progressively estimate these three parameters:

1) Get the $C_{b o t}$ with low-frequency striation structure;

2) Estimate a range of $H$ and $C_{\text {sed }}$ using their relationship with low-frequency striation location;

3) Refine the preliminary estimations to find the best-fit values with high-frequency striation structure.

As demonstrated in former research [35], the use of striation feature for ocean bottom geoacoustic characterization is robust to receiver depth. Here, we only present the result for the hydrophone with an average depth of approximately $19.6 \mathrm{~m}$. Figure 4 gives the calculated spectrogram of run L\#2 using short-time Fourier transform by Matlab [34] with 4096 FFT points, a sliding window of 1s-length and an overlap of $85 \%$. The horizontal line indicates the closest point of approach (CPA) (at about 14:30).

There was an upshift of the spectral lines in the spectrogram, which are due to an abrupt speed change during the navigation. The low-frequency striations during the speed-up are distorted, which are probably due to the complicated source spectra transition between different navigation speeds [36]. However, the overall striation structure is well preserved. The robustness of the striations to navigation speed suggests it is a favourable 


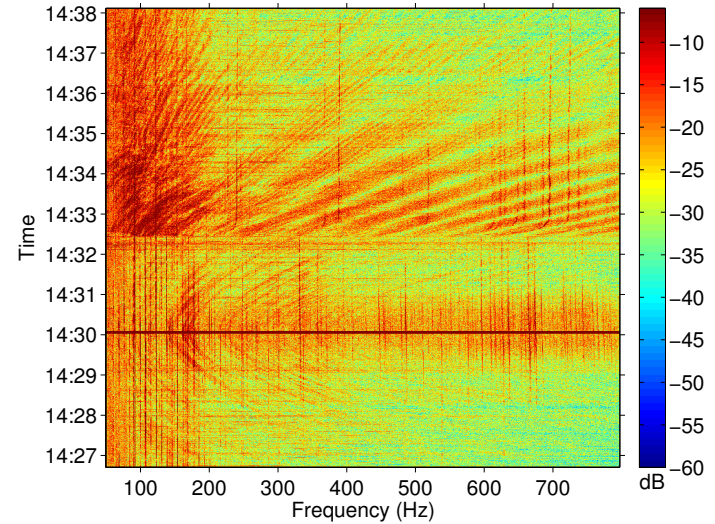

Figure 4: Estimated acoustic pressure spectrogram for the hydrophone with an average depth of $19.6 \mathrm{~m}$ during passive run L\#2.

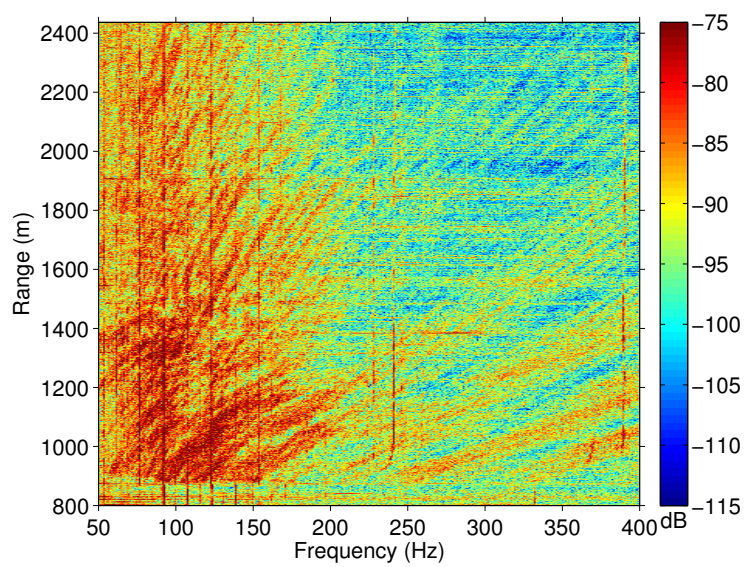

Figure 5: The part of the spectrogram in Fig. 4 of run L\#2 after the speed-up after mapping into range-frequency plane by re-sampling along the time axis after the speed-up.

physical phenomenon for passive acoustic applications.

Moreover, after the speed-up, the spectrogram exhibits more clear striations than before due to the stronger noise. These striations are particularly shown in Fig. 5 after mapping into the frequency-range plane. Figure 6 is the corresponding extracted line structures of Fig. 5, which detects and isolates most striations (the striations in the red box are used to estimate the $C_{b o t}$ in Sec. V-A).

Normally, short-range noise field is expected can better resolve the ocean bottom properties with higher magnitudes than that of far-field. However, according to normal mode theory, more intensive striations appear in the short-range ship noise field than far field due to the contribution of continuous spectrogram. These striations are too intensive to be identified and used for frequency shift calculation in our application for geoacoustic inversion.

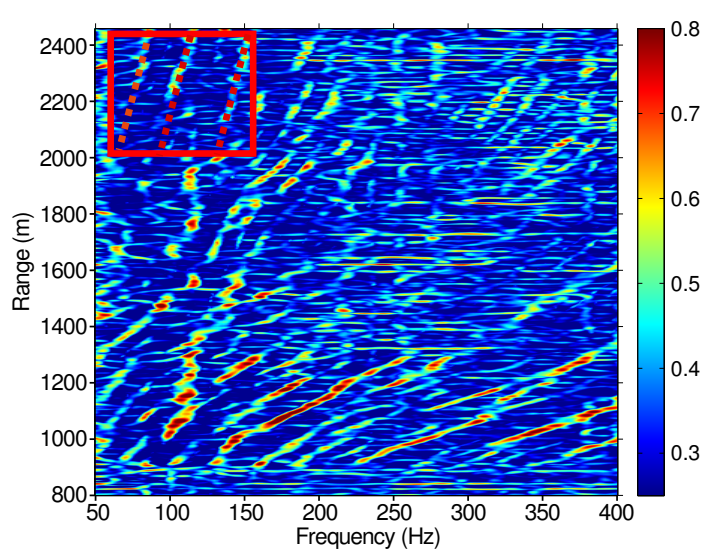

Figure 6: The extracted striation structure of Fig. 5 by the multi-scale line filter.

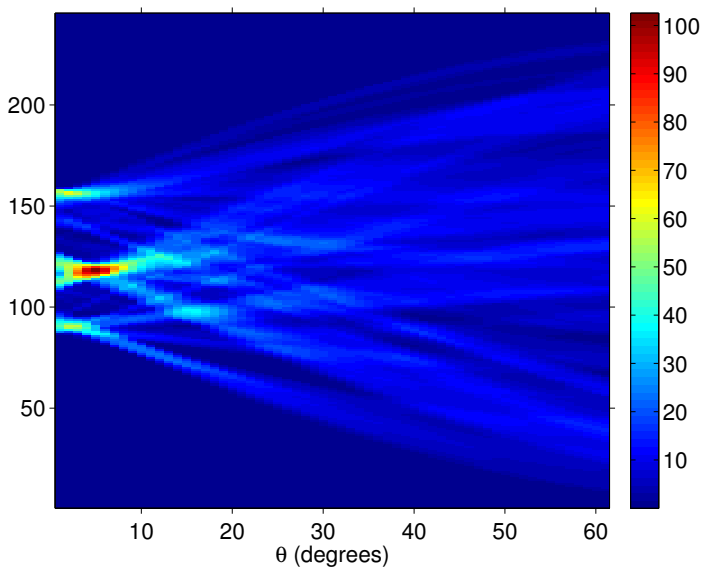

Figure 7: The Radon transform of the red-boxed region in Fig. 6.

\section{Data Processing for ocean Bottom Geoacoustic CHARACTERIZATION}

\section{A. Sub-bottom sound speed estimation}

As shown in Fig. 2, the $H$ and $C_{\text {sed }}$ have little effects on the main low-frequency striation structure. In Fig. 6, the striations in the red box of $[60 \mathrm{~Hz}, 150 \mathrm{~Hz}] \times[2000 \mathrm{~m}, 2400 \mathrm{~m}]$ are used to find the $C_{b o t}$ with some scenario values of $H$ and $C_{\text {sed }}$. The Radon transform [37] is used to extract the striation structure features. The Radon transform of the red-boxed frequencyrange region in Fig. 6 is presented in Fig. 7. The three salient peaks correspond to the three striations.

Based on prior information of the sediment geoacoustic properties of this area, a few pairs of the $C_{\text {sed }}$ and $H$ are selected here to estimate the $C_{b o t}$. The scenario values for the $C_{\text {sed }}$ and $H$ used here are $(1480 \mathrm{~m} / \mathrm{s}, 7.0 \mathrm{~m}),(1470 \mathrm{~m} / \mathrm{s}$, $8.0 \mathrm{~m}),(1480 \mathrm{~m} / \mathrm{s}, 5.0 \mathrm{~m}),(1490 \mathrm{~m} / \mathrm{s}, 6.0 \mathrm{~m})$ and $(1470$ $\mathrm{m} / \mathrm{s}, 10.0 \mathrm{~m}$ ). The correlation coefficients between the Radon transform matrices of the selected striations from the real data and the synthetic data for different $C_{b o t}$ are given in Fig. 8 . The maxima of these curves are all marked by black dots. 


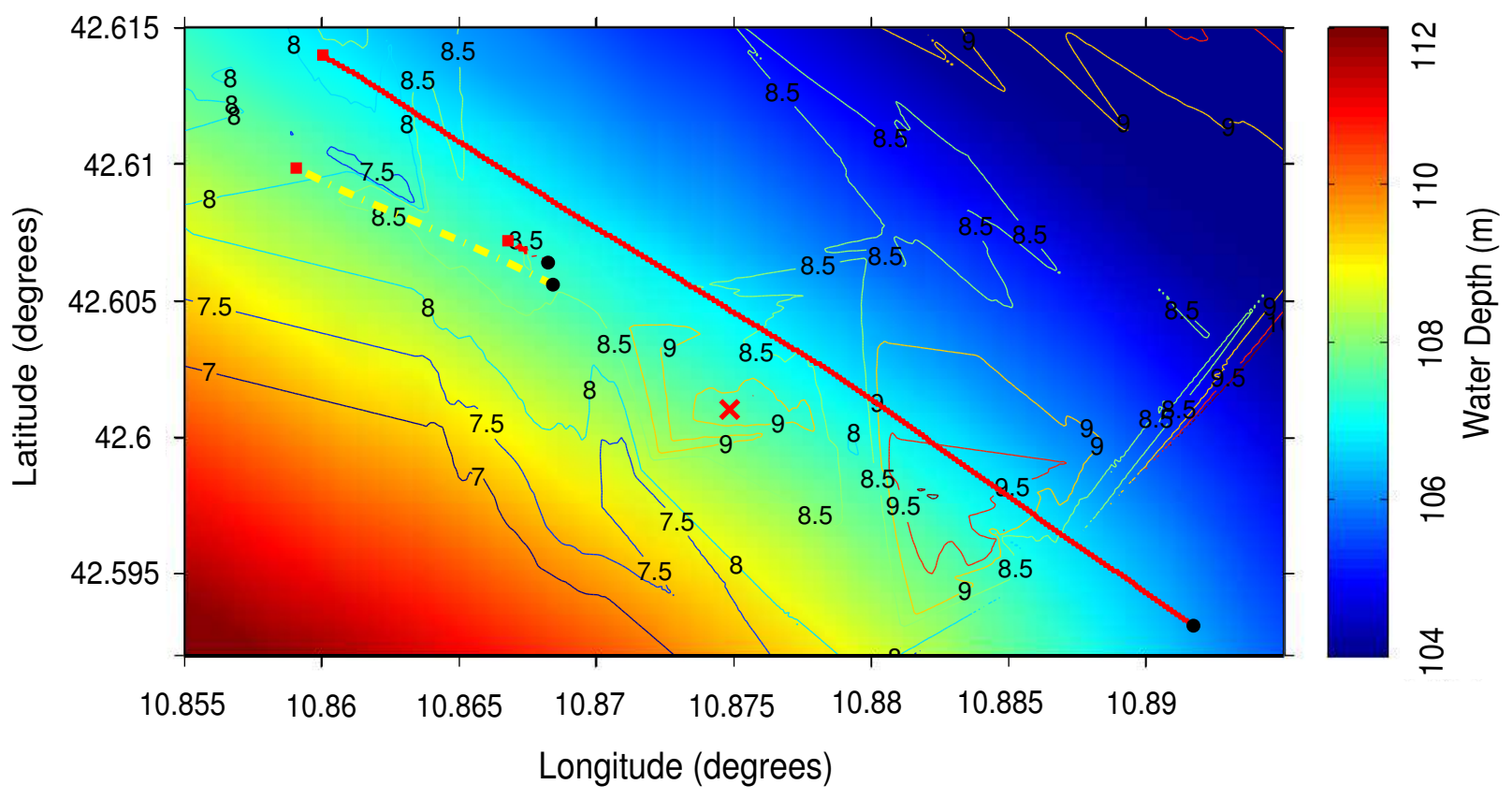

Figure 3: The red solid line indicates the track of passive run conducted by R/V Leonardo (run L\#2) conducted on April 23, 2007. The red dotted line represents the drifting courses of a rubber boat (RHIB) deploying the ocean-acoustic array (OAA) during run L\#2. Contours lines are the thickness of the upper sediment layer reconstructed from the seismic survey. The water depth is illustrated by color scale. An active acoustic measurement was also performed in the same area. The source was deployed from the R/V Leonardo and marked as red cross, the OAA was also deployed from RHIB drifted towards to R/V Leonardo with the course marked by yellow dot line. The start and end points of each measurement are indicated by a square and a dot, respectively.

In spite of the different values for $H$ and $C_{\text {sed }}$, the curves all have their global maximum at $C_{b o t}=1530 \mathrm{~m} / \mathrm{s}$ in Fig. 8 . Therefore, the inverted value for $C_{b o t}$ from the low-frequency striations is $1530 \mathrm{~m} / \mathrm{s}$. There are second peaks at around 1570 $\mathrm{m} / \mathrm{s}$ for each curve, suggesting similar striation structures can be obtained from different ocean geoacoustic parameter sets.

\section{B. Sediment geoacoustic characterization}

In this geoacoustic model, if one selects a reference sediment with $C_{\text {sed }}^{r e f}=1460 \mathrm{~m} / \mathrm{s}$ and $H^{r e f}=0.5 \mathrm{~m}$, the striation location shift $\triangle f$ is almost linear to small sediment perturbations $\left(\triangle C_{\text {sed }}\right.$ and $\triangle H$ ) and their relationship can be interpreted as [35]:

$$
\triangle f=a \triangle C_{\text {sed }}+b \triangle H+c \triangle C_{\text {sed }} \triangle H+d
$$

where $\triangle C_{\text {sed }} \triangle H$ represents the coupled effect of $\triangle C_{\text {sed }}$ and $\triangle H . a, b, c$ and $d$ are constants for specific striation and determined by fitting ambiguity functions in a least-square sense.

As shown in Fig. 6, the striations of real data are often not continuous, which probably due to non-flat ship noise spectra. To use specific striations appeared in different data sets for sediment geoacoustic characterization, one needs to

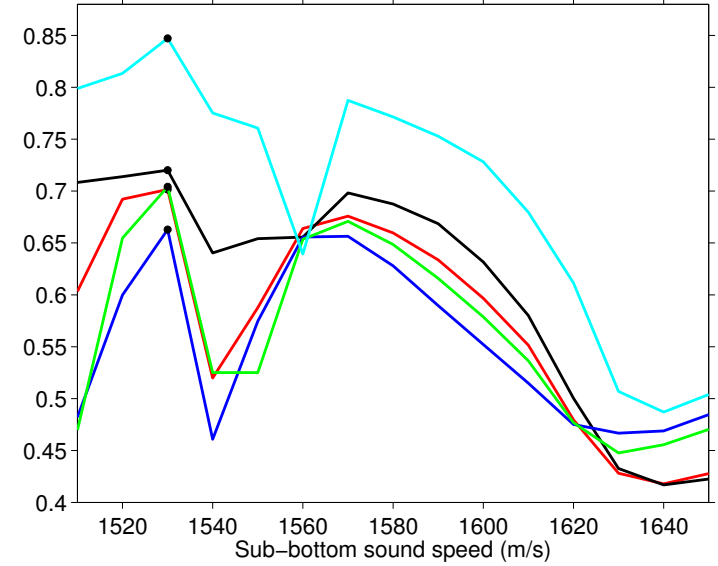

Figure 8: The calculated correlation coefficients between the Radon transform matrices of the data with synthetic data of different $C_{\text {sed }}$ and $H$, red: $1480 \mathrm{~m} / \mathrm{s}, 7.0 \mathrm{~m}$, black: $1470 \mathrm{~m} / \mathrm{s}$, $8.0 \mathrm{~m}$, blue: $1480 \mathrm{~m} / \mathrm{s}, 5.0 \mathrm{~m}$, green: $1490 \mathrm{~m} / \mathrm{s}, 6.0 \mathrm{~m}$, cyan: $1470 \mathrm{~m} / \mathrm{s}, 10.0 \mathrm{~m}$. 

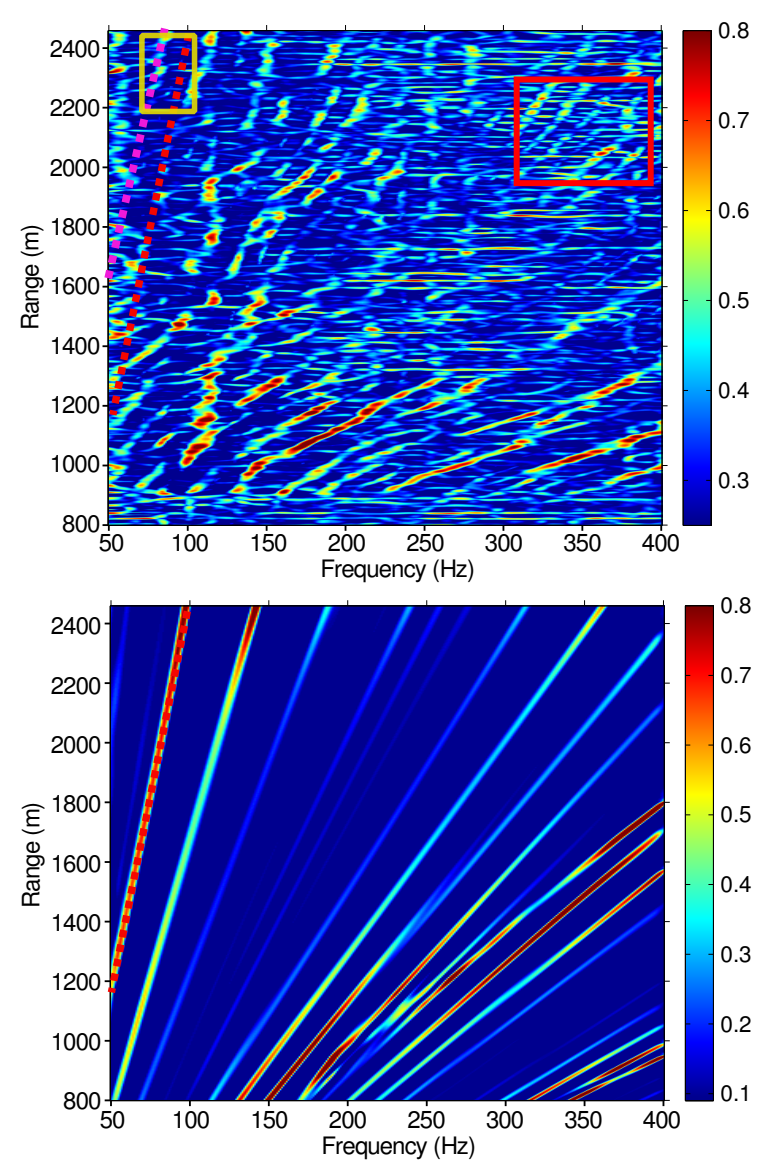

Figure 9: The striations selected for frequency shift calculation and refinement of the first estimate obtained from the yellow box. The striations used to calculate the frequency shift are marked as pink and red dashed lines for real data and synthetic data, respectively. The striations in the new red box are used in Sec. V-B to refine the candidate solutions given by the selected low-frequency striation.

extract the overall effect of environmental perturbation on striation shift for a certain range and frequency intervals. For the frequency-range intervals that contains salient striations selected in this paper, the average values of these constants are $a=0.0093, b=-1.8908, c=0.0204$ and $d=0.2903$ [38]. With these constants, we can estimate the $\triangle C_{\text {sed }}$ and $\triangle H$ for an unknown sediment with respect to reference sediment by matching the predicted $\left(\triangle f^{p r e}\right)$ and measured $\left(\triangle f^{m e a}\right)$ frequency shifts:

$$
\left|\triangle f^{\text {pre }}-\triangle f^{m e a}\right| \leq e r r_{\text {bound }}
$$

where err $_{\text {bound }}$ is the precision.

Eq. 5 gives a range of estimations and need to be refined to find the best solution among them. The high frequency striation's slope and structure are very sensitive to environment [30], whose structure characteristics is used to refine the preliminary estimations.

Through above analysis, for a given $C_{b o t}$, we can estimate

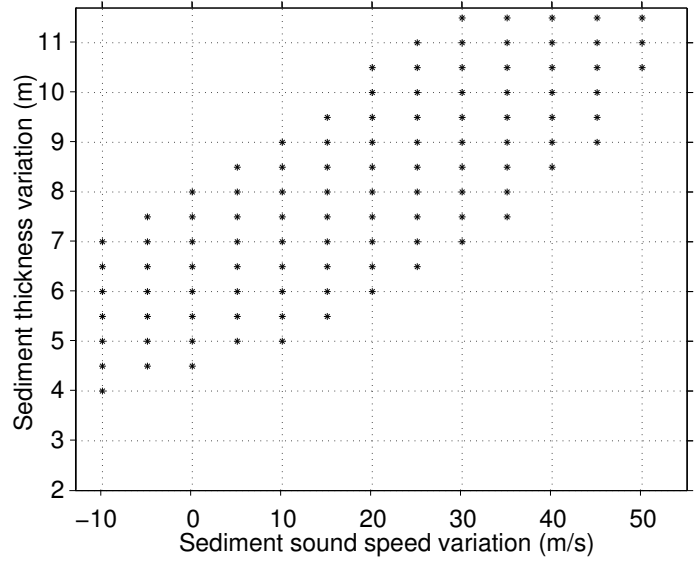

Figure 10: The dominant solutions given by the striation shift compared to that of reference sediment.

the $\triangle C_{\text {sed }}$ and $\triangle H$ with the following steps [35]:

1) Measure the $\triangle f^{m e a}$ of the low-frequency striation of unknown sediment with respect to reference sediment with the $C_{\text {sed }}^{r e f}$ and $H^{r e f}$;

2) Use Eq. 5 to give the likely range of solutions for the $\triangle C_{\text {sed }}$ and $\triangle H$ with respect to the $C_{\text {sed }}^{r e f}$ and $H^{r e f}$;

3) Refine the preliminary estimation with high-frequency striation structure and find the best-fit $\triangle C_{\text {sed }}$ and $\triangle H$;

4) Obtain the $C_{\text {sed }}$ and $H$ of the unknown sediment by summation of the $\triangle C_{\text {sed }}$ and $\triangle H$ with $C_{\text {sed }}^{r e f}$ and $H^{r e f}$, respectively.

Figure 9 shows the extracted line structures of real data and synthetic data for the reference sediment (top sub-figure is a duplication of Fig. 6). They show similar striation structures, especially for low frequency. By observation, the striations of real data (pink-dashed line) and the synthetic data of reference sediment (red-dashed line) in the yellow box are selected for $\triangle f$ calculation. The estimated $\triangle f$ is $-12 \mathrm{~Hz}$, by setting err $r_{\text {bound }}$ to $4 \mathrm{~Hz}$ in Eq. 5 as in [38]. Figure 10 gives a range of estimations for the $\triangle H$ and $\triangle C_{\text {sed }}$ compared to the reference sediment.

To find the best fit-solution from candidate solutions, the CCs between the Radon transform matrices of the red-boxed striations for real data and the candidates are given in Fig. 11. The highest CC yields the best fit for $\triangle H$ and $\triangle C_{\text {sed }}$ are 9.0 $\mathrm{m}$ and $35 \mathrm{~m} / \mathrm{s}$, respectively. Consequently, the $H$ and $C_{\text {sed }}$ along the run L\#2 are obtained by simple summations:

$$
\begin{gathered}
H=H^{\text {ref }}+\triangle H=0.5+9.0=9.5 \mathrm{~m} \\
C=C_{\text {sed }}^{\text {ref }}+\triangle C_{\text {sed }}=1460+35=1495 \mathrm{~m} / \mathrm{s}
\end{gathered}
$$

As described in Sec. IV, the data processed here is the ship noise of run L\#2 after the speed-up toward the end of this run. The corresponding $H$ for this time interval is among $9.0 \mathrm{~m}$ and $9.5 \mathrm{~m}$ (Fig. 3), very close to the $H$ given by the method.

It should be pointed out that, the $C_{\text {sed }}$ given by this method is a bit higher than most active inversion methods [4], [32], [39]. The minor difference may due to the method's 




Figure 11: The correlation coefficients between the Radon transform matrices of high frequency striations inside the red box of Fig. 8 with that of dominant solutions.

insensitivity to the $C_{\text {sed }}$, the presence of the fast thin layer's effect on the acoustic field in the water column [4]. Similar results about the $C_{\text {sed }}$ can also be found in [32] for some active measurements: a higher value was often obtained by high-frequency acoustic data than that of low-frequency.

\section{CONCLUSION}

This paper discusses a cost-effective technique to estimate ocean bottom geoacoustics using ship noise of opportunity. We use a multi-scale line filter to extract and isolate the striations from the broadband ship noise field. For this particular environment, the different frequency-band striations' different sensitivities to the critical ocean bottom parameters $\left(C_{b o t}\right.$, $C_{\text {sed }}$ and $H$ ) are used to progressively estimate these three parameters.

We processed the acoustic data of passive run L\#2 of BP'07 experiment. The inverted results are in good agreement with active inversion results and seismic data in the same area, demonstrating the accuracy of the method for ocean bottom geoacoustic characterization. The current technique can not fully characterize the ocean bottom geoacoustic properties, but can provide initial guess for critical parameters that close to the true values for full-field geoacoustic inversion techniques, e.g., sequential Bayesian filtering technique.

\section{REFERENCES}

[1] E. L. Hamilton, "Geoacoustic modeling of the sea floor," The Journal of the Acoustical Society of America, vol. 68, no. 5, pp. 1313-1340, 1980.

[2] N. Chapman, S. Chin-Bing, D. King, and R. Evans, "Benchmarking geoacoustic inversion methods for range-dependent waveguides," IEEE Journal of Oceanic Engineering, vol. 28, no. 3, pp. 320-330, Jul. 2003.

[3] D. Tollefsen and S. E. Dosso, "Bayesian geoacoustic inversion of ship noise on a horizontal array." The Journal of the Acoustical Society of America, vol. 124, no. 2, pp. 788-795, Aug. 2008.

[4] J.-P. Hermand, "Broad-band geoacoustic inversion in shallow water from waveguide impulse response measurements on a single hydrophone: theory and experimental results," IEEE Journal of Oceanic Engineering, vol. 24, no. 1, pp. 41-66, 1999.
[5] J.-P. Hermand, M. Meyer, M. Asch, and M. Berrada, "Adjoint-based acoustic inversion for the physical characterization of a shallow water environment," The Journal of the Acoustical Society of America, vol. 119, pp. 3860-3871, 2006.

[6] K. D. Heaney, D. D. Sternlicht, A. M. Teranishi, B. Castile, and M. Hamilton, "Active Rapid Geoacoustic Characterization Using a Seismic Survey Source," IEEE Journal of Oceanic Engineering, vol. 29, no. 1, pp. 100-109, Jan. 2004.

[7] M. Siderius, C. H. Harrison, and M. B. Porter, "A passive fathometer technique for imaging seabed layering using ambient noise," The Journal of the Acoustical Society of America, vol. 120, pp. 1315-1323, 2006.

[8] M. J. Buckingham, J. R. Potter, and C. L. Epifanio, "Seeing underwater with background noise," Scientific American, vol. 274, no. 2, pp. 86-90, Feb. 1996.

[9] C. H. Harrison and M. Siderius, "Bottom profiling by correlating beamsteered noise sequences." The Journal of the Acoustical Society of America, vol. 123, no. 3, pp. 1282-1296, Mar. 2008.

[10] D. J. Battle, P. Gerstoft, W. A. Kuperman, W. S. Hodgkiss, and M. Siderius. Geoacoustic inversion of tow-ship noise via near-fieldmatched-field processing. IEEE Journal of Oceanic Engineering, 28(3):454-467, July 2003.

[11] N. R. Chapman, R. M. Dizaji, and R. L. Kirlin. INVERSION OF GEOACOUSTIC MODEL PARAMETERS USING SHIP RADIATED NOISE Cross-relation Matched Field Processor, pages 289-302. Springer, 2006.

[12] K. D. Heaney, D. D. Sternlicht, A. M. Teranishi, B. Castile, and M. Hamilton. Active Rapid Geoacoustic Characterization Using a Seismic Survey Source. IEEE Journal of Oceanic Engineering, 29(1):100109, January 2004.

[13] R. A. Koch and D. P. Knobles. Geoacoustic inversion with ships as sources. The Journal of the Acoustical Society of America, 117(2):626637, 2005.

[14] S. A. Stotts. A robust spatial filtering technique for multisource localization and geoacoustic inversion. The Journal of the Acoustical Society of America, 118(1):139-162, 2005.

[15] C. Park, W. Seong, and P. Gerstoft. Geoacoustic inversion in time domain using ship of opportunity noise recorded on a horizontal towed array. The Journal of the Acoustical Society of America, 117(4):19331941, 2005.

[16] T. C. Yang, K. B. Yoo, and L. T. Fialkowski. Subbottom profiling using a ship towed line array and geoacoustic inversion. The Journal of the Acoustical Society of America, 122(6):3338-3352, 2007.

[17] M. G. Morley, N. R. Chapman, T. McGee, and B. Woolsey. Estimating Geoacoustic Properties of Marine Sediments by Matched Field Inversion Using Ship Noise.

[18] S. Chuprov, "Interference structure of a sound field in a layered ocean," Ocean Acoustics, Current State, pp. 71-91, 1982.

[19] R. Goldhahn, G. Hickman, and J. Krolik, "Waveguide invariant broadband target detection and reverberation estimation." The Journal of the Acoustical Society of America, vol. 124, no. 5, pp. 2841-51, Nov. 2008.

[20] R. Goldhahn, G. Hickman, and J. Krolik, "A waveguide invariant adaptive matched filter for active sonar target depth classification." The Journal of the Acoustical Society of America, vol. 129, no. 4, pp. 18131824, Apr. 2011.

[21] T. C. Yang, "Beam intensity striations and applications," The Journal of the Acoustical Society of America, vol. 113, no. 3, pp. 1342-1352, 2003.

[22] A. Turgut, M. Orr, and D. Rouseff, "Broadband source localization using horizontal-beam acoustic intensity striations," The Journal of the Acoustical Society of America, vol. 127, pp. 73-83, 2010.

[23] E. K. Westwood, "Broadband matched-field source localization," The Journal of the Acoustical Society of America, vol. 91, no. 5, pp. 27772789, 1992.

[24] S. António, S. M. Jesus, and J. Gomes, "Depth and range shift compensation using waveguide invariant properties," 2nd International Conference \& Exhibition on "Underwater Acoustic Measurements: Technologies \& Results", pp. 1315-1320, 2007.

[25] A. F. Frangi, W. J. Niessen, K. L. Vincken, and M. A. Viergever, "Multiscale vessel enhancement filtering," Medical Image Computing and Computer-Assisted Interventation-MICCAI'98, vol. 1496, pp. 130137, 1998.

[26] T. Lindeberg, "Edge detection and ridge detection with automatic scale selection," in Conf. on Comp. Vis. and Pat. Recog, 1996, p. 465.

[27] M. B. Porter, "The KRAKEN Normal Mode Program," 1997. [Online]. Available: http://oalib.hlsresearch.com/Modes/kraken.pdf 
[28] L. M. Brekhovskikh and Y. P. Lysanov, Fundamentals of Ocean Acoustics, 3rd ed. Springer, 2003.

[29] D.M.F. Chapman, , P.D., Ward and , D.D, Ellis. "The effective depth of a Pekeris ocean waveguide, including shear wave effects" The Journal of the Acoustical Society of America, vol. 85, 648-653, 1989.

[30] Q. Y. Ren, J.-P. Hermand, and S. C. Piao, "Space-Frequency Distribution of the Vector Field of Broad-Band Sound in Shallow Water," in OCEANS 10 MTS/IEEE Seattle Conference - Innerspace: A Global Responsibility, 2010.

[31] J.-P. Hermand and J.-C. Le Gac. "Subseafloor geoacoustic characterization in the kilohertz regime with a broadband source and a 4-element receiver array". In Oceans 2008, Quebec City, QC, 2008.

[32] O. Carrière and J.-P. Hermand. "Sequential Bayesian geoacoustic inversion for mobile and compact source-receiver configuration", The Journal of the Acoustical Society of America, (accepted).

[33] K. Siemes, M. Snellen, A.R. Amiri-simkooei, D.G. Simons, and J.-P. Hermand, "Predicting Spatial Variability of Sediment Properties From Hydrographic Data for Geoacoustic Inversion," IEEE Journal of Oceanic Engineering, vol. 35(4), pp. 766-778, 2010.
[34] MATLAB. "version 7.10.0 (R2010a)". The MathWorks Inc., Natick, Massachusetts, 2010

[35] Q. Y. Ren and J.-P. Hermand, "A robust passive interferometry technique for sediment geoacoustic characterization " in in Proc. OCEANS 11 MTS/IEEE Kona Conf, Sept, 2011.

[36] P. T. Arveson and D.J. Vendittis. " Radiated noise characteristics of a modern cargo ship". The Journal of the Acoustical Society of America, vol. 107(1), pp. 118-129, 2000.

[37] G. Beylkin, "Discrete radon transform" IEEE Transactions on Acoustics, Speech and Signal Processing, 35(2): pages 163-172, 1987.

[38] Q. Y. Ren, J.-P. Hermand, and S. C. Piao, "Acoustic interferometry for geoacoustic inversion using broadband ship noise in the Yellow Shark environment," in Proc. Pacic Rim Underwater Acoustics Conference 2011, pp. 85-88, Oct, 2011.

[39] J.-P. Hermand and P. Gerstoft. "Inversion of broad-band multitone acoustic data from the YELLOW SHARK summer experiments". IEEE Journal of Oceanic Engineering, 21(4):324-346, 1996. 